\title{
DECIPHERING PSYCHOLOGICAL-PHYSIOLOGICAL MAPPINGS WHILE DRIVING AND PERFORMING A SECONDARY MEMORY TASK
}

\author{
John K. Lenneman, Jonathon R. Shelley, Richard W. Backs \\ Central Michigan University \\ Mount Pleasant, Michigan, USA \\ E-mail: lenneljk@cmich.edu
}

\begin{abstract}
Summary: An autonomic space model of sympathetic and parasympathetic influences on the heart has been proposed as a method of deciphering psychological-physiological mappings for driving-related tasks. In the current study, we explore the utility of the autonomic space model for deciphering mappings in a driving simulation environment by comparing a single-task driving-only condition to two dual-task, driving-with-a-secondary-workingmemory task conditions. Although limited by a small sample size, the results illustrate the advantages physiological measures can have over performance measures for detecting changes in the psychological process required for drivingrelated task performance. Future research will include a repetition of this same study with more subjects as well the collection of on-the-road autonomic nervous system data.
\end{abstract}

\section{INTRODUCTION}

O'Donnell and Eggemeier (1986) define diagnociticity as the "capability of a technique to discriminate the amount of workload imposed on different operator capacitites or resources." (pp. 42-3). Thus, a physiological measure is diagnostic to the extent that it indexes psycholgical processes, that is, the extent to which the measure exhibits a one-to-one psychologicalphysiological mapping. Backs (2001) proposed that deciphering psychological-physioloical mappings can be improved with an autonomic space model of sympathetic and parasympathetic influences on the heart.

\section{THE MODES OF AUTONOMIC CONTROL}

The heart is dually-innervated by the sympathetic and parasympathetic branches of the autonomic nervous system. Sympathetic activation causes an increase in heart rate, whereas parasympathetic activation causes a decrease in heart rate. Traditionally, activity of these branches was thought to be reciprocal: sympathetic activation was coupled with parasympathetic withdrawal (increased heart rate), and parasympathetic activation was coupled with sympathetic withdrawal (decreased heart rate; Cannon, 1939). Any change in heart rate was always believed to be the result of some change in the reciprocity of the two branches (i.e., one of two modes of autonomic control).

The "Doctrine of Autonimc Space" posits that autonomic nervous system activity is multidimensionally determined instead of reciprocally coupled (Berntson, Cacioppo, \& Quigley, 1991). In addition to being reciprocally coupled, the sympathetic and parasympathetic branches can be non-reciprocally coupled (coactivation or coinhibition) or even uncoupled (change in activity of one branch can occur independent of activity of the other). Thus, instead of a change 
in heart rate being the sum of activation of one branch and withdrawal of the other, eight modes of autonomic control exist (see Figure1).

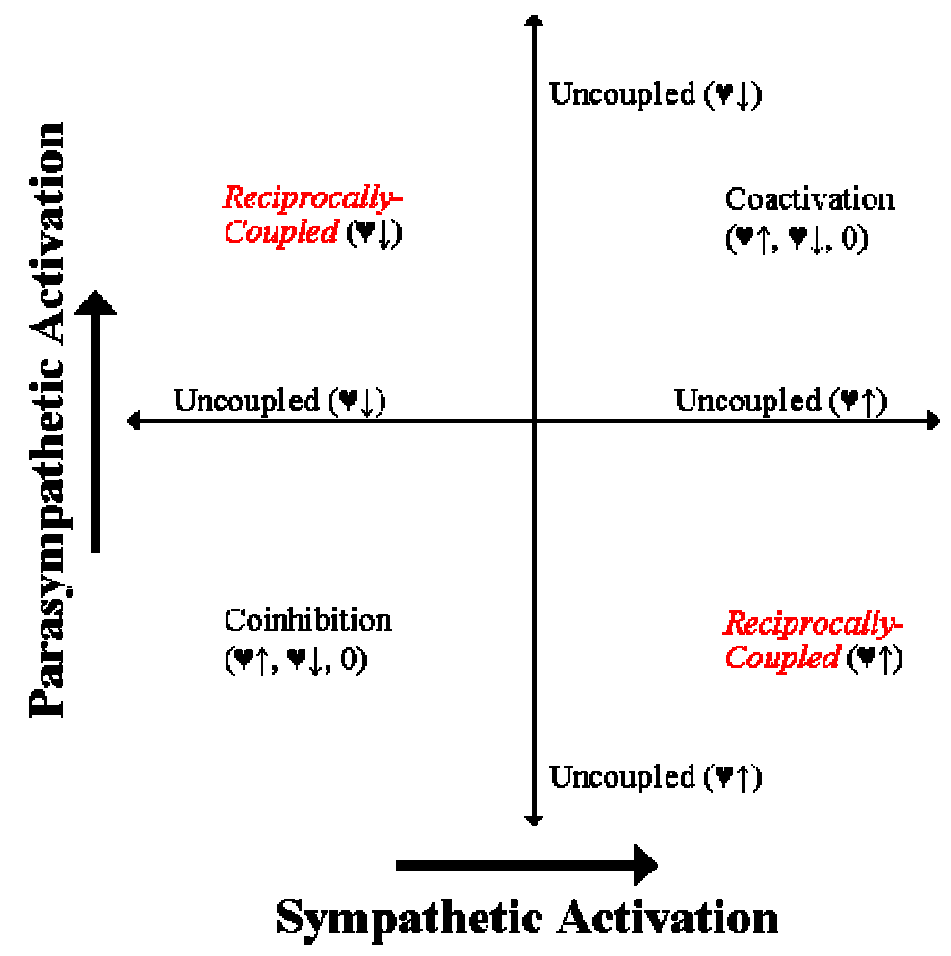

Figure 1. The Eight Modes of Autonomic Control. Traditional coupled modes in italics; $\boldsymbol{\nabla}=$ heart rate; coinhibition and coactivation have multiple responses for heart rate depending on the amount of activation and inhibition of each nervous system.

\section{PURPOSE OF THE STUDY}

Recently, research has demonstrated the utility of autonomic space to decipher psychologicalphysiological mappings while driving (Backs, et al., 2003; Lenneman \& Backs, 2003).

However, the driving task in those studies was a simple single-task driving simulation (i.e., without the presence of any secondary task). The purpose of the present study is to test the utility of the autonomic space model, in addition to the more traditional cardiovascular measures, for deciphering psychological-physiological mappings in a more complex dual-task driving simulation.

\section{METHODS}

Ten subjects ( 9 male, 1 female), who were in good health and not taking any medications that affect the cardiovascular system, participated in the study. Subjects ranged in age from 18 to 31 $($ mean $=20.4)$ years. The subjects participated in two tasks during the study, sometimes concurrently. During one task, participants performed a simulated driving task (they had to steer but velocity was controlled by the computer) using a desktop DriveSafety driving simulator running HyperDrive software (ver. 1.9). The driving environment depicted in the simulation was a straight, two-lane road with no traffic ahead (see Figure 2). Tactile feedback was given to the driver if the vehicle exceeded the left or right edge-line. During the other task, participants 
performed a working memory n-back task presented at one of two levels of difficulty: 0-back and 3-back.

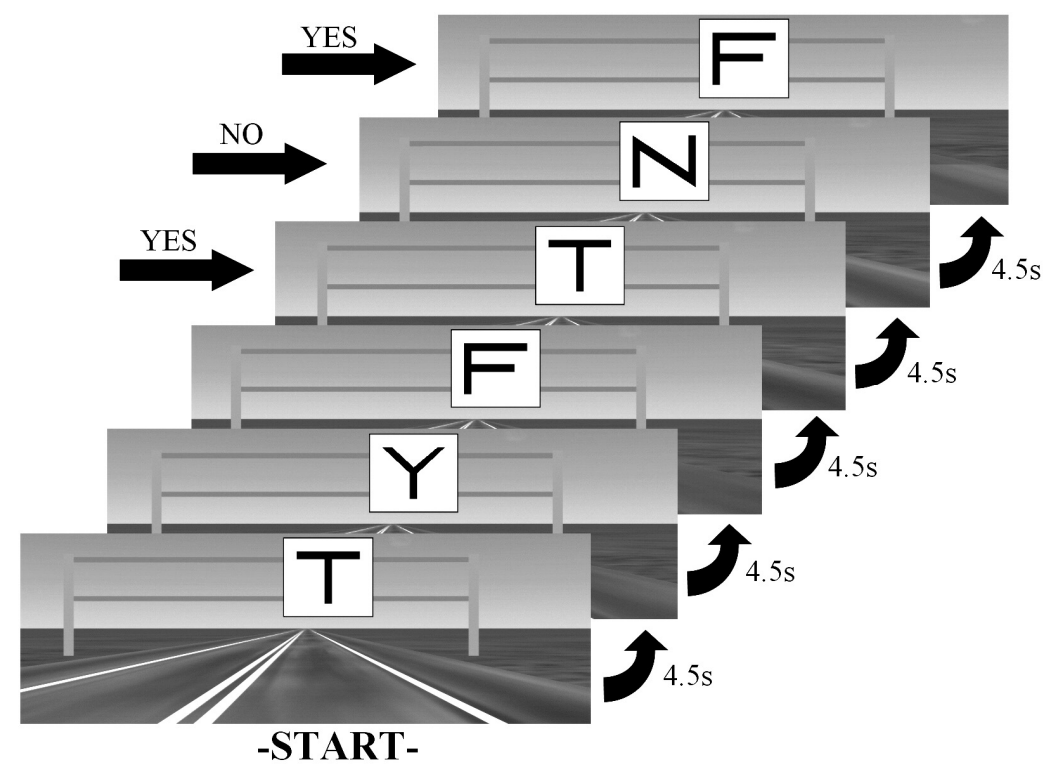

Figure 2. A 3-back task presented on road signs in the simulated environment

An n-back task is a working memory task in which the amount of attentional resources required to perform the task increases as the number of letters to be remembered between the stimulus and target increases. During the 0-back task, the subject was required to specify whether the current letter (the stimulus) was the same as the first letter presented at the beginning of the simulation run (the target). During the 3-back task, the participant was required to specify whether the stimulus matched the letter that was presented three trials previously (the target; see Figure 2). The stimuli for the n-back task were presented on road signs placed every $90 \mathrm{~m}$. Thus, when traveling at $72.4 \mathrm{kph}(45 \mathrm{mph})$, the stimuli were presented to the driver every 4.5 seconds. A total of 53 letters were presented to the subject during each simulation run. During both n-back tasks, the participants signaled whether the stimulus and target matched by pressing one of two buttons on the steering wheel.

During the testing session, five total combinations of the two tasks described above were used in the study: three single-task and two dual-task. For this analysis, we will focus on three of those tasks. In one task, subjects were required to drive the simulation without having to perform the secondary n-back task (single-task driving-only). In two dual-task conditions, subjects drove the simulation while performing either a 0-back or 3-back task. Two trials of each of the three tasks were included in the analysis for a total of six four-minute simulation runs.

Electro- and impedance cardiography was used to obtain noninvasive indices of sympathetic and parasympathetic nervous system activity. The sympathetic index, pre-ejection period (PEP), was obtained from the first derivative of pulsatile changes in transthoracic impedance $(\mathrm{dZ} / \mathrm{dt})$. The parasympathetic index, respiratory sinus arrhythmia (RSA), was measured using high frequency heart rate variability $(0.14-0.40 \mathrm{~Hz})$. PEP and RSA change from resting baseline conditions have been validated as indices of parasympathetic and sympathetic innervation of the heart using pharmacological manipulations (Berntson, et al., 1994; Cacioppo, et al., 1994). The electro- and 
impedance cardiograms were obtained from a Minnesota Impedance Cardiograph Model 304B using a Pentium computer running Mindware Acquisition (Mindware Technologies, Inc.) data acquisition system. Heart period (defined as the time in milliseconds between successive Rpeaks) was used instead of heart rate (number of beats per minute). Heart period, RSA, and PEP were assessed continuously during task performance.

\section{RESULTS}

For reasons of power, an alpha of .10 was used to determine statistical significance. Analysis of physiological measures was performed on difference scores that were computed from a resting baseline condition to task.

\section{Driving Performance}

A 3 (task: driving-only, driving with 0-back, driving with 3-back) x 2 (trial) repeated measures analysis of variance (ANOVA) was conducted for RMS error. The main effect of task $(\mathrm{p}=.120)$, the main effect of trial $(p=.138)$, and the interaction between task and trial $(p=.497)$ were not significant.

\section{Autonomic Space}

A 3 (task: driving only, driving with 0-back, driving with 3-back) x 2 (trial) repeated measures ANOVA was conducted for heart period, PEP and RSA. The main effect of task was significant for heart period, $F(1,9)=15.50, \mathrm{p}=.002$, but not for PEP $(\mathrm{p}=.869)$ or RSA $(\mathrm{p}=.293)$. Heart period decreased (i.e., faster heart rate) significantly from single-task driving-only to dual-task driving with 0-back and dual-task driving with 3-back (see Table 1). The main effect of trial was not significant for heart period $(\mathrm{p}=.394)$, PEP $(\mathrm{p}=.343)$, or RSA $(\mathrm{p}=.642)$.

Table 1. Means and Standard Deviations for the Physiological Changes from Baseline for the Three Tasks

\begin{tabular}{|l|c|c|c|c|c|c|}
\hline \multirow{2}{*}{ Physiological Measure } & \multicolumn{2}{|c|}{ Driving-only } & \multicolumn{2}{c|}{ Driving with 0-back } & \multicolumn{2}{c|}{ Driving with 3-back } \\
\cline { 2 - 6 } & Mean & SD & Mean & SD & Mean & \multicolumn{1}{c|}{ SD } \\
\hline Heart Period (ms) & -28.230 & 6.554 & -58.108 & 8.926 & -85.064 & 13.074 \\
PEP (ms) & -0.068 & 1.972 & -0.666 & 1.626 & -0.936 & 1.890 \\
RSA (ln[ $\left.\left[\mathrm{ms}^{2}\right]\right)$ & -0.434 & 0.164 & -0.538 & 0.185 & -0.712 & 0.244 \\
\hline
\end{tabular}

Analyses of the change from baseline for the three tasks revealed a significant decrease in RSA for driving-only, $F(1,9)=6.99, \mathrm{p}=.027$, driving with 0 -back, $F(1,9)=8.46, \mathrm{p}=.017$, and driving with 3-back, $F(1,9)=8.52, \mathrm{p}=.017$, but no significant change in PEP. In summary, an uncoupled parasympathetic withdrawal mode of control was elicited by driving, but the mode of control did not change across the three tasks. 


\section{DISCUSSION}

O'Donnell and Eggemeier (1986) define sensitivity as the "capability of a technique to detect changes in the amount of workload imposed on task performance" (pp. 42-3). One reason why physiological measures may be more useful to assess changes in mental workload than performance measures is because they may be more sensitive. That is, the physiological measure may change without there being any change in the performance measure (Backs, et al, 1995). Humans who are motivated to perform a task may strive to maintain performance in the face of increasing workload until the point of a catastrophic performance failure. In the current study, there was no significant difference in driving performance across the three tasks. However, the three tasks did elicit significantly different responses in heart period. In particular, the addition of a task that required central/executive processing resources (the n-back task) significantly decreased heart period (i.e., increased heart rate) for both dual-tasks. Thus, in contrast to driving performance, heart period was sensitive to the manipulation of the psychological processes required for task performance. This finding illustrates the real potential to miss changes in psychological processes because of the addition of a secondary task (e.g., navigation) to a primary task such as driving. The use of physiological measures can make up for performance insensitivity; the effects of adding a secondary task may not be reflected in changes in performance (e.g., driving) but may be reflected by changes in physiological measures (e.g., heart period).

In the current study, the only physiological measure to reflect changes in the psychological processes required for task performance was heart period. We believe that this happened for two reasons. First, similar to the situation described above between performance measures and heart period, it is possible that heart period is more sensitive than autonomic space to the changes in the psychological processes required to perform the three tasks. This is certainly possible considering the low number of subjects that participated in the study. Second, the low number of subjects that participated in the study led to low statistical power for the analyses. The baselineto-task analyses illustrate that autonomic space is sensitive to driving-related attentional processes. It is our belief that the collection of more data will result in changes in autonomic space not only from resting baseline, but from driving only to dual-task driving with n-back performance. In turn, not only will heart period be useful for deciphering psychologicalphysiological mappings, but so will autonomic control modes.

Clearly, our findings are limited by a small sample size and, in turn, small power. The logical next step in this line of research is to collect more data. As stated above, we believe that this will result in significant changes in autonomic space and those changes will reflect changes in the psychological processes required for task performance. While the results of this small study suggest that autonomic space may be useful in deciphering psychological-physiological mappings, we believe the results of a larger study will clearly demonstrate the utility of autonomic space for deciphering psychological-physiological mappings in more complex driving situations. Future research will include a repetition of this same study with more subjects, as well as the collection of on-the-road autonomic nervous system data. 


\section{REFERENCES}

Backs, R.W. (2001). An autonomic space approach to the psychophysiological assessment of mental workload. In: P.A. Hancock, \& P.A. Desmond (Eds.), Stress, Workload, and Fatigue. Mahwah, NJ: Lawrence Erlbaum Associates: 279-289.

Backs, R.W., Lenneman, J.K., Wetzel, J.M., \& Green, P. (2003). Cardiac measures of driver workload during simulated driving with and without visual occlusion. Human Factors, 45: 525-538.

Backs, R. W., Ryan, A. M., Wilson, G. F., \& Swain, R. A. (1995). Topographic EEG changes across single-to-dual task performance of mental arithmetic and tracking. Proceedings of the Human Factors and Ergonomics Society 39th Annual Meeting: 953.

Berntson, G.G., Cacioppo, J.T., Binkley, P.F., Uchino, B.N., Quigley, K.S., \& Fieldstone, A. (1994). Autonomic cardiac control, III: Psychological stress and cardiac response as revealed by autonomic blockade. Psychophysiology, 31: 599-608.

Berntson, G.G., Cacioppo, J.T., \& Quigley, K.S. (1991). Autonomic determinism: The modes of autonomic control, the doctrine of autonomic space, and the laws of autonomic constraint. Psychological Review, 98: 459-487.

Cacioppo, J.T., Berntson, G.G., Binkley, P.F., Quigley, K.S., Uchino, B.N., \&Fieldstone, A. (1994). Autonomic cardiac control, II: Noninvasive indices and basal response as revealed by autonomic blockade. Psychophysiology, 31: 586-598.

Cannon, W.B. (1939). The Wisdom of the Body. New York: Norton.

Lenneman, J.K., \& Backs, R.W. (2003). The evolution of autonomic space as a method of mental workload assessment for driving. Proceedings of the Second International Driving Symposium on Human Factors in Driver Assessment, Training, and Vehicle Design . Park City, UT: 125-129.

O’Donnell, R.D., \& Eggemeier, F.T. (1986). Workload assessment methodology. In: K.R. Boff, L. Kaufman, \& J.P. Thomas (Eds.), Handbook of perception and human performance: Vol. II. Cognitive processes and performance. New York: Wiley Interscience: 42-1 - 42-49. 